\title{
Constraints on the extragalactic magnetic fields from the NVSS Faraday rotation measures
}

\author{
M.S. Pshirkov* \\ Sternberg Astronomical Institute, Lomonosov Moscow State University, Universitetsky prospekt \\ 13, 119992, Moscow, Russia \\ Institute for Nuclear Research of the Russian Academy of Sciences, 117312, Moscow, Russia \\ Pushchino Radio Astronomy Observatory, 142290 Pushchino, Russia \\ E-mail: pshirkovesai.msu.ru

\section{P. G. Tinyakov} \\ Université Libre de Bruxelles, Service de Physique Théorique, CP225, 1050, Brussels, Belgium

\section{F. R. Urban} \\ Université Libre de Bruxelles, Service de Physique Théorique, CP225, 1050, Brussels, Belgium
}

\begin{abstract}
We use rotation measures data from the NVSS catalogue to derive new, statistically robust, upper limits on the strength of extragalactic magnetic fields. The extragalactic contribution to the rotation measures for a given field strength and correlation length grows with the distance. Based on the observation that low-luminosity distant radio sources do not exhibit any trend with their redshift, we then constrain extragalactic fields with Mpc coherence length to be below approximately $1.5 \mathrm{nG}$; a field coherent across the entire observable Universe instead is bounded to be below $0.6 \mathrm{nG}$. These limits do not depend on the particular origin of these cosmological fields.
\end{abstract}

The 34th International Cosmic Ray Conference,

30 July- 6 August, 2015

The Hague, The Netherlands

${ }^{*}$ Speaker. 


\section{Introduction}

MFs are already observed at nearly all scales, from planets to clusters of galaxies [1, 2, 3]; still cosmological magnetic fields remain elusive. These MFs could manifest themselves by rotating the plane of polarisation of electromagnetic waves propagating from far away sources to the Earth. In [4] it was demonstrated that Faraday rotation measures (RMs) of distant radio sources of lowluminosity do not show any significant evolution with redshift. Existence of a extragalactic MF (egMF) of certain strength could lead to a quite distinctive change in the distribution of RMs with distance. Thus the strength of such a field could be limited by a comparison of simulated and observed RMs distributions.

The strongest upper limits on the strength, $\hat{B}$, of present-day egMFs come from microwave background observations [5]: $\hat{B} \lesssim 3 \mathrm{nG}$ for a coherence length $l_{c}=1 \mathrm{Mpc}$, these limits apply only to primordial MFs, i.e., fields generated in the very early Universe, while, for instance, cosmological MFs could be generated at later stages by various astrophysical mechanisms (e.g. [6]).

The analysis of RM data produces less restrictive limits: the same $l_{c}=1 \mathrm{Mpc}$ field is bound to have $\hat{B} \lesssim 6 \mathrm{nG}$ [7]. With our method, and thanks to the new available RM data, these limits were improved fourfold or more: we find that egMFs with coherence lengths of $1 \mathrm{Mpc}$ and strengths above $1 \mathrm{nG}$ are highly incompatible with current RM observations; this limit becomes about thrice as strong if the egMF is coherent across the entire Universe.

\section{Method}

Data. The plane of polarisation of a linearly polarised electromagnetic wave, which moves through a magnetised plasma, rotates by an angle $\varphi$ proportional to the square of the wavelength $\lambda$ : $\varphi=\mathrm{RM} \lambda^{2}$, where

$$
\mathrm{RM}=812 \int_{D}^{0} n_{\mathrm{e}} B_{\|} \mathrm{d} l .
$$

where $n_{\mathrm{e}}$ is the density of free electrons measured in $\mathrm{cm}^{-3}, B_{\|}$is the component of the MF parallel to the line of sight (in $\mu \mathrm{G}$ ), and $D$ is the distance to the source in $\mathrm{kpc} ; \mathrm{RM}$ is measured in $\mathrm{rad} / \mathrm{m}^{2}$.

The largest set of RM of extragalactic sources was compiled in [8], using the NRAO VLA Sky Survey (NVSS) data [9]. This set consists of 37,543 sources, 4002 out of them have known redshifts [10]. From the latter sub-set, we accepted the sources with galactic latitude $|b|>20^{\circ}$, $|\mathrm{RM}|<300 \mathrm{rad} \mathrm{m}^{-2}$.

The observed $R M$ could be splitted as $\mathrm{RM}_{\mathrm{obs}}=\mathrm{RM}_{\mathrm{gal}}+\mathrm{RRM}$, where the first term is the contribution of the regular MF of the Milky Way, and the second term stands for "residual RM", and encodes all other sources of RM once the local (regular) MF is subtracted: RM instrinsic to the source, measurement errors, turbulent galactic MF, and egMF (RRMx).

We wanted to disentangle redshift-dependent effects from those intrinsic to sources themselves, thus we computed their luminosity at $1.4 \mathrm{GHz}$ using the most recent spectral indices from [11]. Not all sources have measured spectral indices and that reduced the total number of objects in our set to 3051. The set was split into two groups using a luminosity threshold of $L_{1.4 \mathrm{GHz}}=10^{27.8} \mathrm{~W} \mathrm{~Hz}^{-1}$ : the low luminosity group consists of 2593 sources, while the high luminosity one includes 458 of them. The $\mathrm{RM}_{\mathrm{gal}}$ contribution is subtracted from $\mathrm{RM}_{\mathrm{obs}}$, giving RRMs. 


\begin{tabular}{c|ccccccccc}
$z_{b}$ & 0.15 & 0.35 & 0.7 & 1.3 & 1.65 & 1.95 & 2.25 & 2.6 & 5 \\
\hline$N_{s}$ & 418 & 418 & 501 & 677 & 291 & 137 & 76 & 50 & 25 \\
\hline$\langle|\mathrm{RRM}|\rangle$ & 16.2 & 15.3 & 15.9 & 16.6 & 15.4 & 15.8 & 16.2 & 13.9 & 16.3
\end{tabular}

Table 1: Upper bin redshift boundaries $z_{b}$, numbers of sources in the bin $N_{s}$, and their averages $\langle|\mathrm{RRM}|\rangle$, for the low-power set.

For the lower power group, RRMs do not evolve with redshift and, subsequently, this is the data set we employ in this work. Table 1 summarises this observation.

Simulations. The extragalactic MFs, if present, affect observed RMs [7, 12]: the RRMx grow with redshift. The redshift independent behaviour which we observed instead is thus incompatible with what is expected if an egMF were present.

A model for RRMx needs the properties of the egMF and the electron density $n_{\mathrm{e}}$ to be specified. The latter is assumed to be traced by the observed Lyman- $\alpha(\operatorname{Ly} \alpha)$ forest distribution of neutral hydrogen absorption lines [7, 12]. We use the analytical approximation which was given in $[13,14]$, which is a standard log-normal distribution for the electron overdensity, $\delta_{\mathrm{e}}$, with scale parameter

$$
\sigma_{\mathrm{e}}(z)=0.08+\frac{5.37}{(1+z)}-\frac{4.21}{(1+z)^{2}}+\frac{1.44}{(1+z)^{3}}
$$

and location $\mu_{\mathrm{e}}(z)=-\sigma_{\mathrm{e}}^{2}(z) / 2$ :

$$
P\left(\delta_{\mathrm{e}}\right)=\frac{1}{\sqrt{2 \pi} \sigma_{\mathrm{e}}\left(1+\delta_{\mathrm{e}}\right)} \exp \left\{-\frac{\left[\ln \left(1+\delta_{\mathrm{e}}\right)-\mu_{\mathrm{e}}(z)\right]^{2}}{2 \sigma_{\mathrm{e}}^{2}}\right\} .
$$

This distribution is accurate for fluctuations at the Jeans length scale $\lambda_{J}(z) \simeq 2.3(1+z)^{-3 / 2} \mathrm{Mpc}[13]$ (we adopt $H_{0}=71 \mathrm{~km} / \mathrm{s} / \mathrm{Mpc}$ as the Hubble parameter today and $\Omega_{M}=0.27$ as the total matter density fraction). The actual electron density is finally expressed as $n_{\mathrm{e}}(z)=n_{\mathrm{e}}(0)\left(1+\delta_{\mathrm{e}}\right)(1+z)^{3}$, with $n_{\mathrm{e}}(0)=1.8 \times 10^{-8} \mathrm{~cm}^{-3}$.

The MF in the model is characterised by two parameters: its strength $\hat{B}$ and its coherence length $l_{c}$. In our simulations we test two values for $l_{c}$ : the Jeans length $\lambda_{J}$ and the Hubble size $1 / H_{0}$. At these scales it can be safely assumed that the MF is frozen into the plasma; thus, for spherical overdensities, it will evolve accordingly as $B(z)=\hat{B}\left[n_{\mathrm{e}}(z) / n_{\mathrm{e}}(0)\right]^{2 / 3}$.

We generate a large number of lines of sight in steps of $\lambda_{J}$ up to some given redshift, and we collect RRMx from each step. The electron density is generated at each step sampling the distribution (2.2), also we simulate randomly oriented MF, recalculating its amplitude each time the distance travelled equals a multiple of the correlation length, drawing from a uniform $[-1,1]$ distribution.

The expected theoretical egMF-induced $|\mathrm{RRMx}|$ evolution curves with redshift were obtained, by averaging $10^{5}$ lines of sight out to redshift $z=5$ : the result is given in Fig. 1 for a benchmark MF reference value of $\hat{B}=1 \mathrm{nG}$ : the rapid increase of $|\mathrm{RRMx}|$ with redshift is evident. Also, there is a clear transition in the behaviour of $\operatorname{RRMx}(z)$ roughly localised between $z=0.5$ and $z=1$ : for 


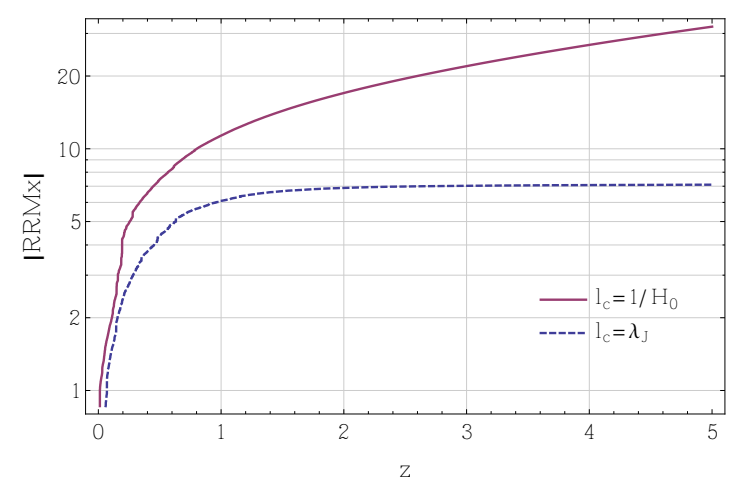

Figure 1: Theoretical egMF-induced $|\mathrm{RRMx}|$ evolution with redshift for $l_{c}=\lambda_{J}$ (blue, dashed), and $l_{c}=$ $1 / H_{0}$ (red, solid), averaged for $10^{5}$ lines of sight.

$l_{c}=1 / H_{0}$ the growth of RM is significantly suppressed at high redshifts, whereas if $l_{c}=\lambda_{J}$ the curve becomes essentially flat.

These simulations could generate only the contribution from the egMF, but there are also contributions from the turbulent Galactic fields, measurement errors, and intrinsic RMs. The two first contributions are dominant and do not depend on redshift; the third one does but it is subdominant. The low-redshift data give information about these redshift-independent contributions, high-redshift portion of the data then could be compared with the simulated distributions for the same set of sources, with the egMF included.

Everything is splitted in three redshift bands: we take the low redshift set to correspond to the bins 1 and 2 of Table 1 , that is, $z=[0,0.35]$, the high redshift set from $z \geq 1.3$ (that is, bins 5 to 9), and a transition set, which was left unused, corresponding to bins 3 and 4 , or $z=[0.35,1.3]$. The three sets contain 836,1178 , and 579 sources, respectively.

The needed simulated distribution of $|\mathrm{RRM}|$ at high- $z$ was constructed as follows (see [15] for details). First, we randomly pick one RRM from the low- $z$ set: this serves as our estimation of the $z$-independent contribution which we can not simulate in our model. We then generate a second batch of RRMx values by simulating 100 lines of sight for each of the sources of the high- $z$ set, that is, a total of 57900 lines of sight. Randomly picked values from both batches (one each) are then incoherently added (that is, each with its own sign) $10^{5}$ times to generate the final theoretical $|\mathrm{RRM}|$ distribution, as a function of the MF strength and coherence length. This distribution in the end is compared with the actual data.

\section{Results}

We compared the two distributions - the data, and the theoretical predicted sample - by means of a Kolmogorov-Smirnov (KS) test. This test allows us to exploit all of the information contained in the distributions, not only first moments in a given redshift bin. Example of this comparison is presented in Fig. 2, it could be easily seen that for $\hat{B}=3 \mathrm{nG}$ and $l_{c}=\lambda_{J}$, the two distributions are statistically incompatible. In Fig. 2 we show an example of the PDFs and CDFs of the two distributions we are comparing: the data and a simulated $|\mathrm{RRM}|$ with $\hat{B}=3 \mathrm{nG}$ and 

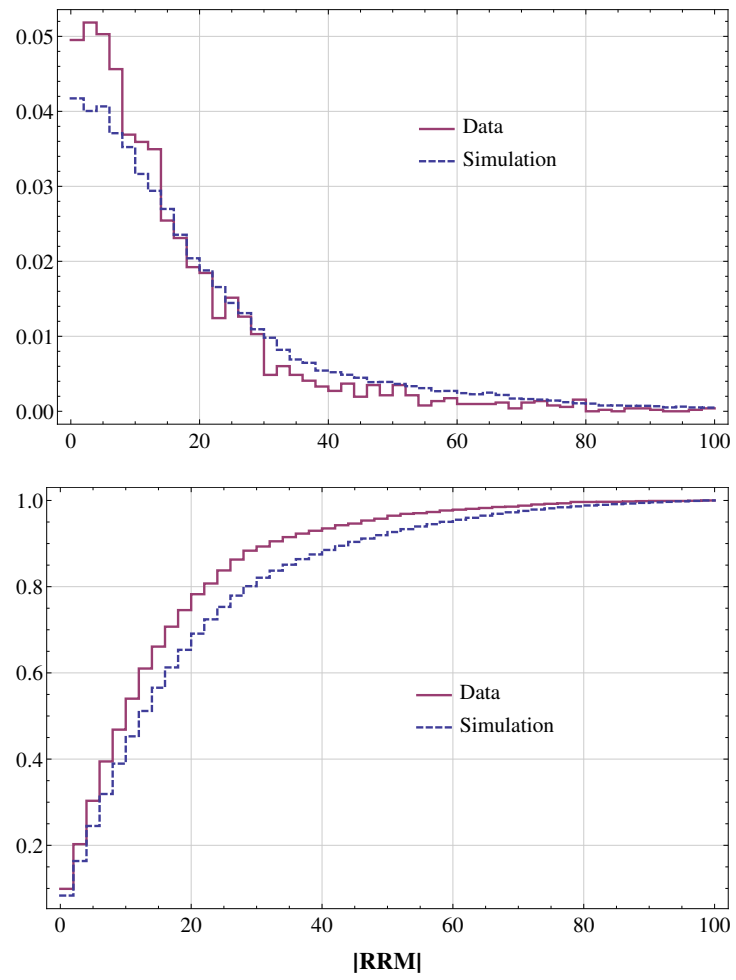

Figure 2: PDF and CDF showing $|\mathrm{RRM}|$ for the data (blue, dashed) and a simulated $|\mathrm{RRM}|$ with $\hat{B}=3 \mathrm{nG}$ and $l_{c}=\lambda_{J}$ (red, solid).

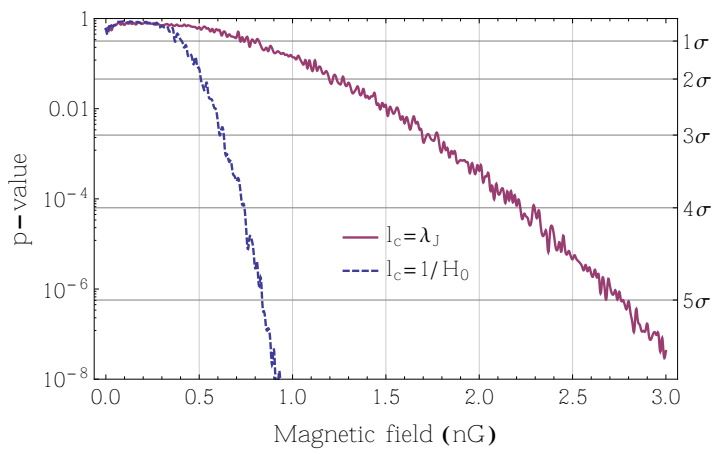

Figure 3: KS tests p-values as a function of $\hat{B}$ for $l_{c}=\lambda_{J}$ (blue, dashed), and $l_{c}=1 / H_{0}$ (red, solid)

$l_{c}=\lambda_{J}$, where it is clear that such a field value is strongly disfavoured - the two distributions are statistically incompatible.

Fig. 3 shows the p-values of the KS tests as a function of $\hat{B}$ for both the $l_{c}=\lambda_{J}$ (blue, dashed), and $l_{c}=1 / H_{0}$ (red, solid) cases. For an egMF with $l_{c}=\lambda_{J}$ the values of $\hat{B}$ corresponding to pvalues of $1 \sigma, 2 \sigma$, and $3 \sigma$ are $\hat{B}=0.65 \mathrm{nG}, \hat{B}=1.2 \mathrm{nG}$, and $\hat{B}=1.7 \mathrm{nG}$, respectively. For the Hubble-scale case the limits are stronger: $3 \sigma$ level is already reached at $\hat{B}=0.6 \mathrm{nG}$. 


\section{Conclusion}

Extragalactic MF with coherence lengths of $1 \mathrm{Mpc}$ can not be stronger than $1.7 \mathrm{nG}$ (at the $3 \sigma$ level), whereas a Hubble-scale egMF is bound to be weaker than about $0.6 \mathrm{nG}$. These limits are obtained using RM data from extragalactic sources, and are valid independently of the origin of these egMF. They are at least a four times improvement over those previously available in the literature, and are now competitive with microwave background ones, which however apply only to primordial fields.

These limits are important to UHECRs propagation: if an egMF with $\hat{B} \simeq 1 \mathrm{nG}$ and $l_{c} \simeq \lambda_{J}$ existed, they would deflect UHECRs significantly. The median deflection for a proton primary of even the highest energy, $10^{20} \mathrm{eV}$, would be around $9 \mathrm{deg}$ when propagating from a distance of $200 \mathrm{Mpc}$.

Acknowledgements. FU and PT are supported by IISN project No. 4.4502 .13 and Belgian Science Policy under IAP VII/37. PT is supported in part by the RFBR grant 13-02-12175-ofi-m. MP are supported by the Russian Science Foundation grant 14-12-01340. MP acknowledges the fellowship of the Dynasty Foundation.

\section{References}

[1] J. P. Vallée, Cosmic magnetic fields - as observed in the Universe, in galactic dynamos, and in the Milky Way, New Astron.Rev. 48 (Sept., 2004) 763-841.

[2] P. P. Kronberg, Extragalactic magnetic fields, Rept.Prog.Phys. 57 (1994) 325-382.

[3] R. Durrer and A. Neronov, Cosmological Magnetic Fields: Their Generation, Evolution and Observation, Astron.Astrophys.Rev. 21 (2013) 62, [arXiv:1303.7121].

[4] M. Pshirkov, P. Tinyakov, and F. Urban, The rotation measures of high luminosity sources as seen from the NVSS, arXiv:1407.3909.

[5] Planck Collaboration, P. A. R. Ade, N. Aghanim, M. Arnaud, F. Arroja, M. Ashdown, J. Aumont, C. Baccigalupi, M. Ballardini, A. J. Banday, and et al., Planck 2015 results. XIX. Constraints on primordial magnetic fields, ArXiv e-prints (Feb., 2015) [arXiv: 1502.0159].

[6] P. P. Kronberg, Q. W. Dufton, H. Li, and S. A. Colgate, Magnetic Energy of the Intergalactic Medium from Galactic Black Holes, ApJ 560 (Oct., 2001) 178-186, [a stro-ph/ 0106281 ].

[7] P. Blasi, S. Burles, and A. V. Olinto, Cosmological magnetic fields limits in an inhomogeneous universe, Astrophys.J. 514 (1999) L79-L82, [astro-ph/9812487].

[8] A. Taylor, J. Stil, and C. Sunstrum, A Rotation Measure Image of the Sky, Astrophys.J. 702 (2009) 1230 .

[9] J. J. Condon, W. Cotton, E. Greisen, Q. Yin, R. Perley, et al., The NRAO VLA Sky survey, Astron.J. 115 (1998) 1693-1716.

[10] A. M. Hammond, T. Robishaw, and B. M. Gaensler, A New Catalog of Faraday Rotation Measures and Redshifts for Extragalactic Radio Sources, arXiv:1209.1438 [astro-ph.CO] (Sept., 2012).

[11] J. Farnes, B. Gaensler, and E. Carretti, A Broadband Polarization Catalog of Extragalactic Radio Sources, Astrophys.J.Suppl. 212 (2014) 15, [arXiv: 1403.2391 ]. 
[12] T. Akahori and D. Ryu, Faraday Rotation Measure due to the Intergalactic Magnetic Field II: the Cosmological Contribution, Astrophys.J. 738 (2011) 134, [arXiv:1107.0142].

[13] H. Bi and A. F. Davidsen, Evolution of structure in the intergalactic medium and the nature of the ly-alpha forest, Astrophys.J. 479 (1997) 523, [a stro-ph/9611062].

[14] P. Coles and B. Jones, A Lognormal model for the cosmological mass distribution, Mon.Not.Roy.Astron.Soc. 248 (1991) 1-13.

[15] M. S. Pshirkov, P. G. Tinyakov, and F. R. Urban, New limits on extragalactic magnetic fields from rotation measures, ArXiv e-prints (Apr., 2015) [arXiv: 1504.0654 ]. 